\title{
Clostridium difficile fecal toxin level is associated with disease severity and prognosis
}

United European Gastroenterology Journal 2018, Vol. 6(5) 773-780 (C) Author(s) 2017 Reprints and permissions: sagepub.co.uk/journalsPermissions.nav DOI: $10.1177 / 2050640617750809$ journals.sagepub.com/home/ueg

\author{
Nathaniel A Cohen ${ }^{1}$, Tamar Miller ${ }^{2}$, Wasef Na'aminh ${ }^{2}$, Keren Hod ${ }^{3}$, \\ Amos Adler ${ }^{4}$, Daniel Cohen ${ }^{5}$, Hanan Guzner-Gur ${ }^{1}$, Erwin Santo ${ }^{6}$, \\ Zamir Halpern ${ }^{6}$, Yehuda Carmeli ${ }^{2}$ and Nitsan Maharshak ${ }^{6,7}$
}

SAGE

\begin{abstract}
Background: Antibiotic-associated colitis caused by Clostridium difficile (C. difficile) is the most common cause of hospitalacquired diarrhea. The pathogenesis of $C$. difficile colitis is mediated by bacterial toxins. $C$. difficile infection (CDI) severity may be determined by the fecal level of these toxins.

Objective: The objective of this article is to determine whether fecal $C$. difficile toxin (CDT) levels are associated with disease severity and prognosis.

Methods: A cross-sectional study of patients admitted with CDI in a tertiary center between 2011 and 2015 was conducted. Fecal CDT levels were determined by quantitative ELISA. Severe CDI was defined as a leukocyte count of $>15 \times 10^{3}$ cells/ $\mu$, creatinine levels that deteriorated by $>1.5$ times the baseline level, or albumin levels $<3 \mathrm{~g} / \mathrm{dl}$.

Results: Seventy-three patients were recruited for this study. Patients with severe CDI ( $n=47)$ had significantly higher toxin levels compared to patients with mild to moderate CDI $(n=26)(651 \mathrm{ng} / \mathrm{ml}$ (IQR 138-3200) versus 164 ng/ml (IQR 55.2-400.1), respectively; $p=0.001)$. A high toxin level $(>2500 \mathrm{ng} / \mathrm{ml}$ ) was associated with an increased mortality rate (odds ratio 11.8 ; $95 \%$ confidence interval $2.5-56)$.

Conclusions: The fecal CDT level is associated with disease severity and mortality rate. Measuring CDT levels may be an objective and accurate way to define the severity of CDI.
\end{abstract}

\section{Keywords}

Infectious diarrhea, infectious colitis, antibiotics, Clostridium difficile

Received: 9 October 2017; accepted: 25 November 2017

\section{Key summary}

- Clostridium difficile infection is the most common cause of antibiotic-associated diarrhea.

- It is a major cause of morbidity and mortality among affected patients.

- Fecal Clostridium difficile toxin (CDT) level correlates with disease severity and confers an increased risk of 30-day mortality.

- Measuring fecal CDT level may allow for better risk stratification and aid in determining patient prognosis.

\footnotetext{
${ }^{1}$ Department of Internal Medicine “B," Tel Aviv Sourasky Medical Center, Tel Aviv, Israel

${ }^{2}$ Department of Epidemiology, Tel Aviv Sourasky Medical Center, Tel Aviv, Israel

${ }^{3}$ Research Division, Assuta Medical Center, Tel Aviv, Israel

${ }^{4}$ Department of Clinical Microbiology, Tel Aviv Sourasky Medical Center, Tel Aviv, Israel

${ }^{5}$ School of Public Health, Tel Aviv University, Tel Aviv, Israel

${ }^{6}$ Department of Gastroenterology and Liver Diseases, Tel Aviv Sourasky Medical Center, Tel Aviv, Israel
}

${ }^{7}$ Bacteriotherapy Clinic, Department of Gastroenterology and Liver Diseases, Tel Aviv Sourasky Medical Center, Tel Aviv, Israel

All authors are affiliated with the Sackler Faculty of Medicine, Tel Aviv University, Tel Aviv, Israel.

Corresponding author:

Nitsan Maharshak, Bacteriotherapy Clinic, Department of Gastroenterology and Liver Diseases, Tel Aviv Sourasky Medical Center, 6 Weizmann St, Tel Aviv 64239, Israel. Email: nitsanm@tlvmc.gov.il 


\section{Introduction}

Antibiotic-associated colitis caused by Clostridium difficile ( $C$. difficile) is the most common cause of hospital-acquired diarrhea. The number of cases doubled from 2000 to 2009, and it is a major cause of morbidity and mortality within the elderly hospitalized patient population in the United States. ${ }^{1} C$. difficile infection (CDI) is mediated by toxins that cause colon injury and inflammation. ${ }^{2}$ The widespread usage of antibiotic therapy, which disrupts the intestinal microbial flora and allows colonization of $C$. difficile, is well recognized as the major risk factor in the spread of this disease, ${ }^{3}$ along with multiple additional risk factors. ${ }^{4}$ It is not entirely clear what determines disease severity, and increasing age and the use of exacerbating antibiotics have been purported to play a role. ${ }^{5,6}$ C. difficile toxin (CDT) levels may also affect disease severity. ${ }^{2}$ This is supported by the observation that patients with negative toxin immunoassay but with positive polymerase chain reaction for the toxin genes have milder disease compared to patients with a measurable toxin level by immunoassay. ${ }^{7,8}$ It is therefore plausible that a quantitative analysis of fecal toxin level may reflect disease severity in patients with immunoassay-positive results. Indeed, it has previously been shown that CDT levels correlate with abdominal pain and diarrhea frequency, ${ }^{9}$ but no correlation between CDT levels and accepted disease severity parameters ${ }^{10}$ or patient prognosis has been previously demonstrated.

Our study aimed to investigate whether fecal CDT levels correlate with standard and objective measures of disease severity, and whether fecal CDT levels can predict patient outcome.

\section{Materials and methods}

\section{Study population}

Stool samples were collected, upon suspicion of CDI, as part of the routine investigation of diarrheal disease in patients admitted at the Tel Aviv Medical Center during the years 2011-2015. Patients were included if they suffered from diarrhea and tested positive for $C$. difficile with an immunoassay test ( $C$. Diff Quik Check $^{\circledR}$, Tech-Lab, USA) and if there was sufficient quantity of frozen fecal samples ( $>50 \mathrm{mg}$ of stool) for toxin analysis. Epidemiological, clinical, and laboratory data were collected for all patients. The study protocol conforms to the ethical guidelines of the 1975 Declaration of Helsinki as reflected in a prior approval by the institution's human research committee, the Helsinki committee, approval number 0528-10TLV (date of approval: January 18, 2011).

\section{Definitions}

Disease severity was defined according to the guidelines of the Society for Healthcare Epidemiology of America (SHEA) and the Infectious Diseases Society of America $(\text { IDSA })^{10}$ as follows: mild to moderate disease $=$ leukocytosis $<15,000$ cells $/ \mu 1$ and creatinine $<1.5$ times the premorbid level; severe disease $=$ leukocytosis $>15,000$ cells $/ \mu 1$ or serum creatinine $>1.5$ times the premorbid level. Severe disease was also defined as a serum albumin level of $<3 \mathrm{~g} / \mathrm{dl}$ at the time of active infection. ${ }^{11}$ The Charlson comorbidity index was used to assess comorbidities. $^{12}$

\section{Fecal toxin level}

C. difficile-positive fecal samples stored at $-80^{\circ} \mathrm{C}$ were thawed to room temperature in order to determine the fecal toxin level. The fecal matter was thawed only at the time of analysis in order to minimize the natural degradation of the CDTs. ${ }^{13}$ Fifty $\mathrm{mg}$ of feces was homogenized with $200 \mu \mathrm{l}$ of diluent buffer and centrifuged $(5000 \times g$ for $10 \mathrm{~min})$ to remove particulate matter.

\section{Test procedure}

The fecal toxin level was quantified according to the manufacturer's instructions (C. Diff Tox A/B $\mathrm{II}^{\mathrm{TM}}$, Tech-Lab, USA), and 96-well plates were used. Two wells were used as negative control (containing diluent buffer), one well as positive control (supplied with the kit), then predetermined standards with known toxin concentration ( $\mathrm{ng} / \mathrm{ml})$ (Calbiochem, Merck Millipore, USA) and diluted specimen (supernatant). The optical density (OD) was measured at a dual wavelength of $450 \mathrm{~nm} / 620 \mathrm{~nm}$ on a microplate enzymelinked immunosorbent assay reader.

\section{Interpretation of results}

A standard curve was created for the toxin concentration by plotting the mean absorbance (OD) against a known toxin concentration $(\mathrm{ng} / \mathrm{ml})$ within the linear range in order to quantify the assay results. Samples with ODs outside the linear range were diluted and multiplied by the dilution factor.

\section{Statistics}

All data were summarized and displayed as the mean \pm standard deviation (SD) for normally distributed continuous variables, as median interquartile range (IQR) for non-normally distributed continuous variables, and as the number of patients plus the percentage 
in each group for categorical variables. The independent $t$-test, Mann-Whitney test and chi-square statistics were used for comparisons between study groups for demographic variables, clinical characteristics, and health disease status. The Spearman correlation was used to assess correlations between disease severity as defined by leukocyte count, deterioration in creatinine and serum albumin levels and fecal CDT levels. A multivariate logistic regression was performed to investigate the relationship between mortality and a set of prognostic factors (toxin levels, Charlson comorbidity index scores, and leukocyte counts), adjusting for several potential confounders, such as current chemotherapy treatment, gender, inflammatory bowel disease (IBD), and use of steroids, proton pump inhibitors (PPIs), and antibiotics. The level of significance used for all analyses was two tailed and set at $p<0.05$. The SPSS statistical package (Version 22, IBM Inc, Chicago, IL, USA) was used for all statistical analyses.

\section{Results}

A total of 73 patients (mean age $69 \pm 17.7$ years, $60 \%$ females, $n=44$ ) who had diarrhea and tested positive for CDT by an immunoassay for $C$. difficile antigen and for CDT toxin (Tech-Lab, USA) were included in the study.

\section{CDT toxin level as a marker of disease severity}

Patients were classified as having mild to moderate or severe disease according to their leukocyte counts or deterioration in the baseline creatinine or serum albumin levels. Table 1 details the demographic, clinical, and disease status characteristics of the groups. The mild-to-moderate group consisted of 26 patients $(36 \%)$ and the severe group consisted of 47 patients $(64 \%)$.

The fecal CDT levels were significantly higher in patients who were classified as having severe disease than those with mild-to-moderate disease $(651 \mathrm{ng} / \mathrm{ml}$ (IQR 138-3200) versus $164 \mathrm{ng} / \mathrm{ml}$ (IQR 55.2-400.1), respectively; $p=0.001$ ) (Figure 1(a)).

We next examined the association between the CDT fecal levels and each of the disease severity subgroups, defined by either leukocyte count or deterioration in baseline creatinine or serum albumin level. Interestingly, the fecal CDT level was significantly higher in severe disease compared to mild-to-moderate disease only when defined by the leukocyte count (i.e., $>15 \times 10^{3}$ cells $\left./ \mu \mathrm{l}\right) \quad(2174.3 \mathrm{ng} / \mathrm{ml} \quad$ (IQR $157.5-$ 4209) vs $167 \mathrm{ng} / \mathrm{ml}$ (IQR 55.6-475), respectively; $p<0.0001$ ). Furthermore, the fecal CDT level showed significant correlation with the leukocyte count $(r=0.515, p<0.001)$ but not with deterioration in the creatinine or serum albumin levels (Figure 1). A receiver operating characteristic curve analysis revealed that a fecal CDT level of $1575 \mathrm{ng} / \mathrm{ml}$ reflected a specificity of $100 \%$ and a sensitivity of $45 \%$ for severe disease (as defined by leukocyte count) (Figure 2).

\section{Increased fecal CDT level is associated with mortality}

Thirteen $(17.8 \%)$ patients died within 30 days of CDI diagnosis. There were no significant demographic or clinical differences between those who died and those who survived except for increased chemotherapy exposure among the former patients $(p=0.004)$ (Supplementary table 1). Fecal CDT levels were significantly increased among patients who died within 30 days post-diagnosis compared to those who survived (2676.3 ng/ml (IQR 200.6-5202) versus $182.3 \mathrm{ng} / \mathrm{ml}$ (IQR 69.7-672.3), respectively; $p=0.008$ ). These results are further highlighted by a Kaplan-Meier survival chart that shows a significantly decreased survival in those patients with a higher toxin level $(p=0.0006)$ (Figures 2 and 3). Moreover, in patients with fecal CDT level $>2500 \mathrm{ng} / \mathrm{ml}$, mortality rate was significantly higher $(p=0.003)$ (Figure 4$)$ and was associated with a death rate of $47 \%(n=7)$ within 30 days of CDI diagnosis.

The fecal CDT level was a predictor of mortality $(p=0.008)$ in a multivariate logistic regression analysis when adjusted for Charlson comorbidity index scores, sex, IBD, and usage of PPIs, antibiotics, and steroids. Patients with a fecal CDT level of $>2500 \mathrm{ng} / \mathrm{ml}$ had an odds ratio (OR) of 11.8 (95\% confidence interval (CI) 2.5-56) of dying within 30 days of CDI diagnosis, in contrast to a nonsignificant OR of 1.08 (95\% CI 0.99 1.18 ) in patients with a leukocyte count $>15 \times 10^{3}$ cells/ $\mu 1$ (Table 2). Patients who received chemotherapy $(n=8)$ had a significantly greater mortality risk $(\mathrm{OR}=15.4,95 \%$ CI 2.5-97.8) when adjusted for these same variables. Interestingly, the CDT level in the latter patient group was significantly higher compared to patients with no chemotherapy exposure $(4702.9 \mathrm{ng} /$ $\mathrm{ml}$ (IQR 164.7-6119.2) versus 240.2 ng/ml (IQR 79$745)$, respectively; $p=0.03$ ), indicating a synergistic effect between these two factors. Indeed, $100 \%(n=4)$ patients with both fecal CDT levels above $2500 \mathrm{ng} / \mathrm{ml}$ and a recent chemotherapy treatment died as opposed to a mortality rate of $15 \%(n=9)$ for those with either one or none of those two factors $(n=69, p=0.001)$. When patients who received chemotherapy were excluded from the analysis, there was still a trend toward higher fecal CDT levels in those who died compared to those who survived $(660 \mathrm{ng} / \mathrm{ml}$ (IQR 1913130) versus $182 \mathrm{ng} / \mathrm{ml}$ (IQR 68-667), respectively; $p=0.06)$. 


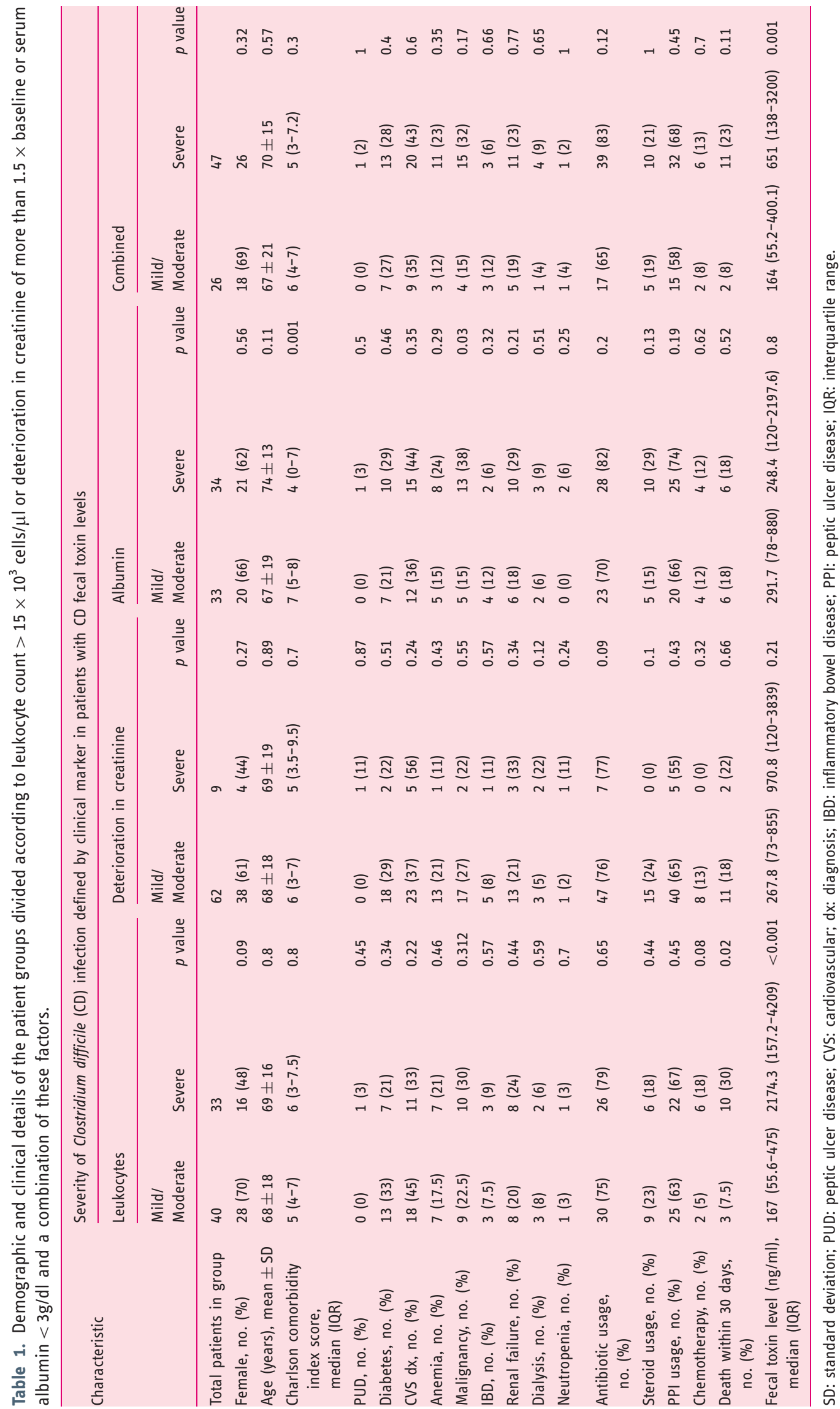






Figure 1. Correlation plots detailing the relationship between fecal $C D$ toxin level and markers of disease severity A) leucocyte count ( $>15,000$ cells/uL), B) deterioration in creatinine level ( $>1.5 \times$ baseline) and C) serum albumin ( $<3 \mathrm{gr} / \mathrm{dl})$.

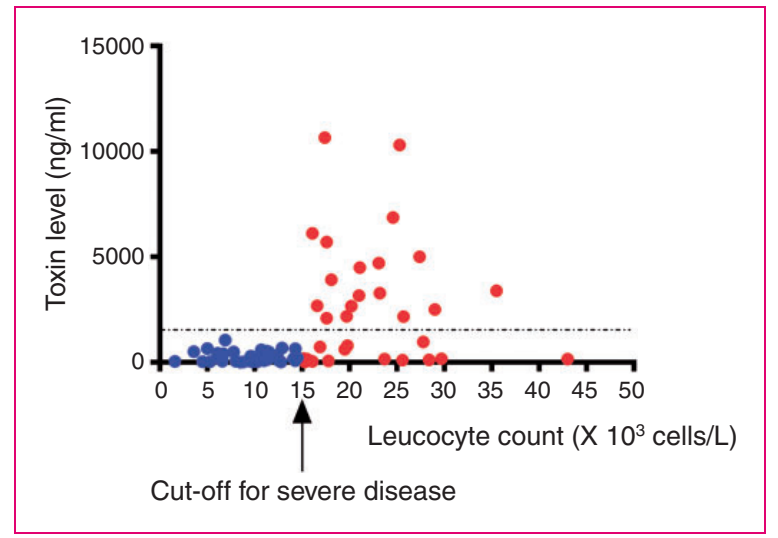

Figure 2. Scatter plot showing that a toxin level of $1575 \mathrm{ng} / \mathrm{ml}$ was associated with $100 \%$ specificity for severe disease (as defined by leucocyte count).

\section{Discussion}

The results of this cross-sectional study revealed an association between the fecal CDT level and CDI disease severity and prognosis. We also found that the fecal CDT level is a good predictor of mortality. Two earlier studies had investigated the association between fecal CDT level and disease severity, ${ }^{9,14}$ but neither looked into its relation with the standard measures of disease severity (i.e., leukocyte count and a deterioration of creatinine or serum albumin levels) nor with mortality.

In this study, fecal CDT level was significantly higher in patients with severe disease compared to those with mild to moderate disease. This was even more apparent when disease severity was defined solely by an increased leukocyte count $(>15,000$ cells $/ \mu 1)$. Furthermore, the fecal CDT level correlated directly to the leukocyte count, further supporting the notion that the fecal CDT level is important in determining disease severity. Importantly, there were no differences in age, sex, comorbidities, chemotherapy treatment, or in steroid, antibiotic, or PPI use between the groups.

A previous investigation of the relationship between the fecal CDT level and disease severity found a trend toward higher fecal CDT levels in patients with severe disease. ${ }^{9}$ That study, however, defined severity in clinical terms (i.e., number of stools per day, abdominal pain, and fever) not routinely used to define disease severity. The current study is the first to describe the association between fecal CDT level and CDI severity based on an objective and accepted definition of disease severity. ${ }^{10,11,15}$ Using this definition avoids the difficulties in quantifying the number of stools per day and the subjective manner of describing abdominal pain.

Currently, accepted measures of disease severity are a high leukocyte count, deterioration in serum creatinine levels, and low serum albumin levels. ${ }^{10}$ There are 


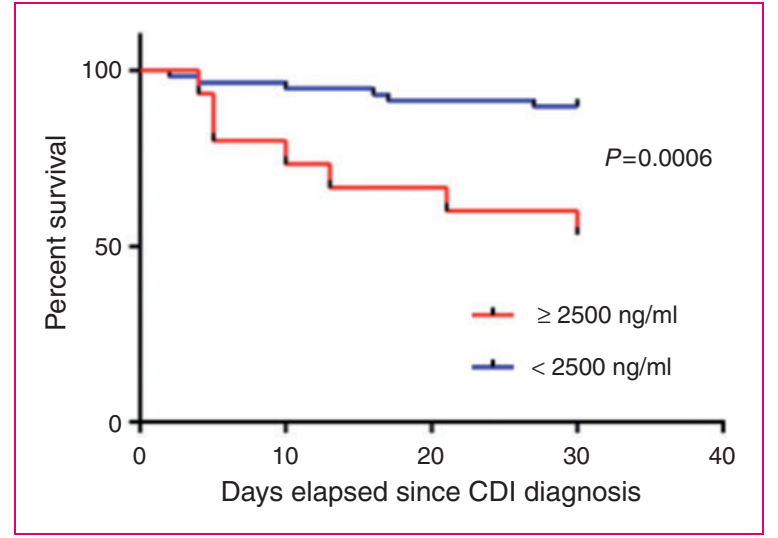

Figure 3. Kaplan-Meier plot showing the significant difference in 30 day mortality between patients with a CD toxin level above $2500 \mathrm{ng} / \mathrm{ml}$ and those with a CD toxin level below this cut off.

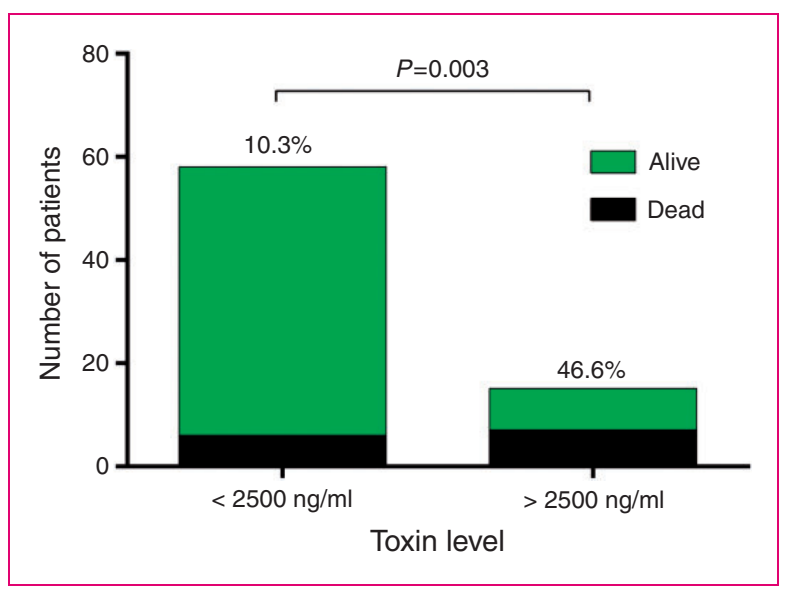

Figure 4. Death rate was significantly higher in patients with a toxin level of greater than $2500 \mathrm{ng} / \mathrm{ml}$.

inherent caveats using these measures in particular patient groups such as those with neutropenia or with numerous sources of a raised leukocyte count, patients with chronic kidney disease and chronically ill patients with a low albumin level at baseline. These factors may lead to either over- or underestimation of CDI severity. In such patients other measures of disease severity are required and fecal CDT levels may assist in risk-stratifying these patients. In the current study, the fecal CDT level could diagnose severe CDI with $100 \%$ specificity. As such, the measuring of fecal CDT levels may help in decision making with regard to implementing more intensive treatment regimens in complicated patients.

An important finding is a positive association between fecal CDT levels and mortality. It is striking the fecal toxin level upon CDI diagnosis was significantly higher than among patients who died compared to those who survived. Patients with a fecal CDT level of $>2500 \mathrm{ng} / \mathrm{ml}$ had an OR risk of $11.8(95 \%$ CI 2.5-56) of dying within 30 days of CDI diagnosis
Table 2. Adjusted odds ratios for 30 -day mortality by clinical feature.

\begin{tabular}{ll}
\hline & $\begin{array}{l}\text { Adjusted for Charlson } \\
\text { comorbidity index } \\
\text { score, IBD, antibiotic } \\
\text { use, PPI use, steroid } \\
\text { use, and sex }\end{array}$ \\
Predictive variables & $11.8(2.5-56.1)$ \\
\hline Toxin level $>2500 \mathrm{ng} / \mathrm{ml}(95 \% \mathrm{CI})$ & $15.4(2.4-98.7)$ \\
Chemotherapy treatment $(95 \% \mathrm{Cl})$ & $1.08(0.93-1.18)$ \\
$\begin{array}{l}\text { Leukocyte count }>15 \times 10^{3} \mathrm{cells} / \mu \mathrm{l} \\
(95 \% \mathrm{Cl})\end{array}$ & \\
\hline
\end{tabular}

IBD: inflammatory bowel disease; PPI: proton pump inhibitor; $95 \% \mathrm{CI}$ : 95\% confidence interval.

after adjustment for potential confounders. It was previously shown that risk factors for complicated CDI and mortality included age, comorbidities, serum albumin level, leukocytosis, increased serum creatinine levels, and a $C$. difficile ribotype. ${ }^{16}$ A direct relationship between the fecal CDT level and mortality has not been described before and it suggests that measuring fecal CDT levels may provide a means for good risk stratification in CDI patients and perhaps should complement the other determinants of severity, that is, leukocyte count, deterioration in creatinine levels, and low serum albumin level.

It is noteworthy that chemotherapy was also associated with mortality in this setting. During the study, eight patients were being actively treated with chemotherapy, and five of them died within 30 days of CDI and the fecal toxin level was $>2500 \mathrm{ng} / \mathrm{mg}$ in four of those five. This may be indicative of a strong synergistic effect of chemotherapy and high toxin levels on CDI mortality. While chemotherapy is a risk factor for CDI, ${ }^{17,18}$ it had not been associated with increased CDI severity or associated mortality. ${ }^{5}$ Despite the small numbers of patients in our current study, the high mortality rate in this patient population should promote further studies to determine whether this result is reproducible and, if so, whether more aggressive treatment of CDI can reduce the mortality rate. It is not clear whether an increased toxin level in this group of patients is a marker of immunosuppression caused by chemotherapy and unrelated to the increased death rate or whether it is the cause of the increased death rate in these patients. It was noted that when patients receiving chemotherapy were excluded, there was still a trend toward higher toxin levels in patients who died, supporting the importance of the CDT level in the pathogenesis and prognosis of these patients. Prospective studies are required to provide better answers to this question.

All the patients in our cohort were admitted to a tertiary care hospital, which uses acceptable treatment proto$\operatorname{cols}^{19}$ that include early initiation of antibiotic therapy (metronidazole, vancomycin, or a combination) shortly 
after CDI diagnosis (Supplementary table 2). Nevertheless, the mortality rates of CDI were high and current measurements of disease severity were not predictive of mortality, thus highlighting the need for a better and more objective definition of disease severity and prognosis. Admission fecal CDT levels of $>2500 \mathrm{ng} / \mathrm{ml}$ were associated with a high mortality rate, and they should guide the clinician to initiate more aggressive and more effective treatment methods, such as early initiation of vancomycin, a combination of metronidazole and vancomycin, or early fecal microbial transplantation. Furthermore, these results also provide a rationale for the investigation of treatments that directly target fecal CDT as described in numerous recent studies. ${ }^{20-22}$

This study has several limitations that bear mention. There was a lack of an association analysis of toxin level and clinical severity indices that were not included owing to the unreliability clinical data collection, particularly the number of bowel movements per day, as this relies on patient memory/awareness and/or accurate determination by the medical staff. This unreliability in data collection also made determining the therapeutic efficacy and clinical improvement difficult to assess and thus the end point of 30-day mortality alone was used to determine outcome. In addition, although we know that the most common strain of $C$. difficile in our hospital is the hr-02 strain, ${ }^{23}$ it would be an important addition to the data to perform a strain analysis, given that certain strains (e.g., ribotype 078) are associated with more severe disease. ${ }^{24}$

\section{Conclusion}

This study shows that $C$. difficile fecal toxin level is associated with increased disease severity and further, very high levels of fecal toxin may predict poorer outcomes and increased short-term mortality in these patients. Fecal CDT level provides a more objective and direct measure of CDI severity than the currently used methods especially considering the multiple comorbidities affecting this patient cohort. Perhaps, using fecal CDT level as a risk stratification tool, more timely and aggressive therapy can be initiated. This study also provides further insight into the pathophysiology of CDI. Finally, although the results of this study are promising, further studies surrounding the prognostic value of $C$. difficile fecal toxin levels with regards to morbidity, mortality, and relapse rates are required.

\section{Declaration of conflicting interests}

None declared.

\section{Funding}

This research received no specific grant from any funding agency in the public, commercial, or not-for-profit sectors.

\section{Ethics approval}

The study protocol conforms to the ethical guidelines of the 1975 Declaration of Helsinki as reflected in a prior approval by the Tel Aviv Medical Center's human research committee and the Helsinki committee (approval number 0528-10-TLV; date of approval: 18 January 2011).

\section{Informed consent}

Patients with laboratory confirmed CDI were asked to sign an informed consent in order to be included in the study.

\section{Acknowledgment}

The authors thank Esther Eshkol for her assistance with the professional English editing of this manuscript.

\section{References}

1. Lessa FC, Gould CV and McDonald LC. Current status of Clostridium difficile infection epidemiology. Clin Infect Dis 2012; 55(Suppl 2): S65-S70.

2. Mahida YR, Makh S, Hyde S, et al. Effect of Clostridium difficile toxin $\mathrm{A}$ on human intestinal epithelial cells: Induction of interleukin 8 production and apoptosis after cell detachment. Gut 1996; 38: 337-347.

3. Hensgens MP, Goorhuis A, Dekkers OM, et al. Time interval of increased risk for Clostridium difficile infection after exposure to antibiotics. $J$ Antimicrob Chemother 2012; 67: 742-748.

4. Janarthanan S, Ditah I, Adler DG, et al. Clostridium difficile-associated diarrhea and proton pump inhibitor therapy: A meta-analysis. Am J Gastroenterol 2012; 107 : 1001-1010.

5. Henrich TJ, Krakower D, Bitton A, et al. Clinical risk factors for severe Clostridium difficile-associated disease. Emerg Infect Dis 2009; 15: 415-422.

6. Manek K, Williams V, Callery S, et al. Reducing the risk of severe complications among patients with Clostridium difficile infection. Can J Gastroenterol 2011; 25: 368-372.

7. Yoldaş Ö, Altındiş M, Cufalı D, et al. A diagnostic algorithm for the detection of Clostridium difficile-associated diarrhea. Balkan Med J 2016; 33: 80-86.

8. Polage CR, Gyorke CE, Kennedy MA, et al. Overdiagnosis of Clostridium difficile infection in the molecular test era. JAMA Intern Med 2015; 175: 1792-1801.

9. Akerlund T, Svenungsson B, Lagergren A, et al. Correlation of disease severity with fecal toxin levels in patients with Clostridium difficile-associated diarrhea and distribution of PCR ribotypes and toxin yields in vitro of corresponding isolates. J Clin Microbiol 2006; 44: 353-358.

10. Cohen SH, Gerding DN, Johnson S, et al. Clinical practice guidelines for Clostridium difficile infection in adults: 2010 update by the Society for Healthcare Epidemiology of America (SHEA) and the Infectious Diseases Society of America (IDSA). Infect Control Hosp Epidemiol 2010; 31: 431-455. 
11. Surawicz CM, Brandt LJ, Binion DG, et al. Guidelines for diagnosis, treatment, and prevention of Clostridium difficile infections. Am J Gastroenterol 2013; 108: 478-498; quiz 499.

12. Charlson M, Szatrowski TP, Peterson J, et al. Validation of a combined comorbidity index. J Clin Epidemiol 1994; 47: $1245-1251$.

13. Freeman $\mathbf{J}$ and Wilcox MH. The effects of storage conditions on viability of Clostridium difficile vegetative cells and spores and toxin activity in human faeces. $J$ Clin Pathol 2003; 56: 126-128.

14. Burdon DW, George RH, Mogg GA, et al. Faecal toxin and severity of antibiotic-associated pseudomembranous colitis. J Clin Pathol 1981; 34: 548-551.

15. Iv EC, Iii EC and Johnson DA. Clinical update for the diagnosis and treatment of Clostridium difficile infection. World J Gastrointest Pharmacol Ther 2014; 5: 1-26.

16. Abou Chakra CN, Pepin J, Sirard S, et al. Risk factors for recurrence, complications and mortality in Clostridium difficile infection: A systematic review. PloS One 2014; 9: e98400.

17. Husain A, Aptaker L, Spriggs DR, et al. Gastrointestinal toxicity and Clostridium difficile diarrhea in patients treated with paclitaxel-containing chemotherapy regimens. Gynecol Oncol 1998; 71: 104-107.

18. Nielson H, Daugaard G, Tvede M, et al. High prevalence of Clostridium difficile diarrhoea during intensive chemotherapy for germ cell cancer. Br J Cancer 1992; 66: 666-667.

19. Cohen NA, Ben Ami R, Guzner-Gur H, et al. Fecal microbiota transplantation for Clostridium difficileassociated diarrhea. Isr Med Assoc J 2015; 17: 510-514.

20. Bender KO, Garland M, Ferreyra JA, et al. A smallmolecule antivirulence agent for treating Clostridium difficile infection. Sci Transl Med 2015; 7: 306ra148.

21. Esposito G, Nobile N, Gigli S, et al. Rifaximin improves Clostridium difficile toxin A-induced toxicity in Caco-2 cells by the PXR-dependent TLR4/MyD88/NF- $\kappa$ B pathway. Front Pharmacol 2016; 7: 120.

22. Gigli S, Seguella L, Pesce M, et al. Cannabidiol restores intestinal barrier dysfunction and inhibits the apoptotic process induced by Clostridium difficile toxin A in Caco-2 cells. United European Gastroenterol J 2017; 5: 1108-1115.

23. Adler A, Miller-Roll T, Bradenstein R, et al. A national survey of the molecular epidemiology of Clostridium difficile in Israel: The dissemination of the ribotype 027 strain with reduced susceptibility to vancomycin and metronidazole. Diagn Microbiol Infect Dis 2015; 83: 21-24.

24. Walker AS, Eyre DW, Wyllie DH, et al. Relationship between bacterial strain type, host biomarkers, and mortality in Clostridium difficile infection. Clin Infect Dis 2013; 56: 1589-1600. 